gen unter anderem der Medien und von supranationalen Institutionen zugeschrieben als auch ganz prominent den Parlamenten selbst.

In publizistischer Hinsicht ist es schade, dass der Band keinen resümierenden Beitrag enthält, der die Machtfrage anhand der Texte noch einmal reflektiert. Ebenso fehlt ein Verzeichnis der Autoren. Außerdem hätte die redaktionelle Bearbeitung etwas mehr Sorgfalt vertragen, um zum Beispiel fast leere Seiten aufgrund von Tabellenpositionierungen zu vermeiden. Es zeigt sich überdies, dass es Beiträgen in Sammelbänden nicht gut tut, wenn sie auf umfangreichen Monographien (Kranenpohl, Köppl und vor allem Sebaldt) beruhen, da sie so zu Kurzzusammenfassungen werden, ohne einen für diese Art von Text eigentlich notwendigen argumentativen Bogen spannen zu können.

Franziska Carstensen

\title{
Seiteneinsteiger im Bundestag: Karriereweg und politische Arbeit
}

\section{Bailer, Stefanie, Peter Meißner, Tamaki Ohmura und Peter Selb: Seiteneinsteiger im Deutschen Bundestag, VS Verlag für Sozialwissenschaften, Wiesbaden 2013, 151 Seiten, € 29,99.}

Wer anders ist, erregt Aufsehen. Das gilt natürlich auch in der Politik. Dort werden die so genannten Seiteneinsteiger zumeist kritisch beäugt. Von Quereinsteigern, -denkern und -köpfen ist dann die Rede, von Mutigen, Andersdenkenden und Greenhorns, von parteipolitischen Frischlingen, bunten Vögeln oder Fachleuten ohne parteilichen Stallgeruch: Die Bezeichnungen sind vielfältig. Gerade die Berichterstattung richtet sich häufig auf neue Namen und Köpfe in der Politik. Doch das (mediale) Interesse an Seiteneinsteigern in der Politik war bis vor einigen Jahren deutlich größer als die wissenschaftliche Aufbereitung. Neben dem Politikwissenschaftler Dietrich Herzog, der sich von 1975 an mit politischen Karrieren beschäftigte ${ }^{1}$, sind erst in der jüngeren Vergangenheit einige Werke hinzu gekommen. ${ }^{2}$ Dazu zählt auch die Untersuchung des Autorenteams von der ETH Zürich und der Universität Konstanz, die auf Anregung des Studentenforums des Tönissteiner Kreises und zusammen mit ihm entstand. „Seiteneinsteiger im Deutschen Bundestag“ lautet der Titel, doch das Buch bietet - trotz des geringen Umfangs - mehr: Es ist eine interessante Analyse der Karrierewege aller Bundestagsabgeordneten der 17. Wahlperiode, also von 2009 bis 2013.

Entscheidend für das Gelingen der Studie ist, dass die Autoren die Schwierigkeit erkennen, Seiteneinsteiger in dem heterogenen Feld der Parlamentarier zu identifizieren. Dieser

1 Vgl. unter anderem Dietrich Herzog, Politische Karrieren - Selektion und Professionalisierung politischer Führungsgruppen, Opladen 1975.

2 Speziell mit Seiteneinsteigern in Deutschland haben sich folgende Studien befasst: Robert Lorenz I Matthias Micus (Hrsg.), Seiteneinsteiger. Unkonventionelle Politiker-Karrieren in der Parteiendemokratie. Göttinger Studien zur Parteienforschung, Wiesbaden 2009; Moritz Küpper, Politik kann man lernen. Politische Seiteneinsteiger in Deutschland, Halle (Saale) 2013; allgemeiner zu Politischen Karrieren in Deutschland: Anwar Syed Ali, Karrierewege und Rekrutierungsmuster bei Regierungsmitgliedern auf Bundesebene von 1949-2002, Halle (Saale) 2003; Andreas K. Gruber, Der Weg nach ganz oben. Karriereverläufe deutscher Spitzenpolitiker, Wiesbaden 2009; speziell für Seiteneinsteiger in der österreichischen Politik: Armin Wolf, Promi-Politik. Prominente Quereinsteiger in der österreichischen Politik, Innsbruck / Wien 2005. 
begegnen sie nicht mit der klassischen Negativ-Auswahl - Seiteneinsteiger haben nicht die „Ochsentour“ durch politische Ämter und die Partei gemacht -, die auf einem Zeitpunkt abstellt, sondern berücksichtigen vielmehr alle Karriereschritte sowie deren Abfolge und Dauer. Dazu wird die so genannte Sequenzanalyse verwendet, bei der zum Beispiel ParteiEintritt, lokales Parteiamt, öffentliches Amt auf lokaler, Landes- oder Bundes-Ebene der Abgeordneten codiert und dann in den Kontext von beispielsweise „Alter“ und auch „Wahl in den Bundestag“ gestellt wird. Die biografischen Daten sind „Kürschners Volkshandbuch Deutscher Bundestag“ entnommen. An dieser Stelle wird jedoch nicht deutlich, wie sehr die Autoren auf diese von den Parlamentariern freiwillig und nicht immer vollständig geleisteten Angaben vertrauen. Zudem setzen sie darauf, dass, sollte ein Abschnitt nicht klar als beendet gekennzeichnet sein, dieser nur bis zur nächsten Tätigkeit lief, und übersehen so das (häufige) Phänomen der Ämterkumulation. Ungeachtet dessen ist das Ergebnis dieses Ansatzes durchaus beeindruckend und führt zu sechs identifizierbaren Karriereverläufen: „Seiteneinsteiger“ (mit einem Anteil von zehn Prozent), „Ostdeutsche“ (zwölf), „Local Heroes“ (17), „Junge Karrieristen“ (25), „Parteiochsentour“ (elf) und „Normalos“ (25). Der Anteil der Seiteneinsteiger liegt dabei in der Spanne der bisherigen Forschungen. Auch die gefundenen Charakteristika, dass diese eher älter sind, wenn sie ihren Parteien beitreten, eher auf Bundes- als auf Regionalebene einsteigen und am häufigsten in kleinen Fraktionen präsent sind, bestätigen die bisherigen Erkenntnisse.

Der große Mehrwert dieser Studie liegt darin, dass die Autoren nicht bei dieser Bilanz verharren, sondern fragen, welche Auswirkung der jeweilige Karriereweg auf die politische Arbeit hat. Dies wird beispielsweise an der Verteilung der Bundestagsausschüsse, dem Repräsentationsverständnis der Abgeordneten sowie deren Abstimmungsverhalten gemessen. Grundlage für diesen zweiten Teil waren Umfragen unter Parlamentariern, rund 70 leitfadengestützte Experteninterviews sowie Daten zum Abstimmungsverhalten. Der Fokus liegt dabei auf der Gruppe der Seiteneinsteiger und führt zu hochinteressanten Ergebnissen (S. 130): So wird in den Fraktionsführungen vor allem die fachliche Expertise der Seiteneinsteiger geschätzt; diese orientieren sich bei ihrem Selbstverständnis eher an den Bürgern allgemein als an den Wählern in ihrem Wahlkreis. Dazu passt, dass Seiteneinsteiger in wahlkreisorientierten Ausschüssen unterdurchschnittlich, in wichtigen Ausschüssen aber durchschnittlich oft vertreten sind. Ansonsten stimmen sie eher parteikonform ab. Diese Merkmale führen über eine soziodemografische Bestandsaufnahme der Gruppe der Seiteneinsteiger hinaus. Diesbezügliche Befunde sind neu und stellen eine Fortführung der bisherigen Seiteneinsteiger-Forschung dar.

Trotz vieler Schaubilder und Tabellen ist das Buch gut lesbar, bietet aufgrund seiner Struktur aber auch die Möglichkeit, einzelne Komplexe kurz nachzuschlagen oder sich Impulse zu holen. Dies liegt auch an der Zusammenstellung plakativer Interview-Aussagen am Ende des Bandes, die zweierlei bewirken: Zum einen stellen sie die wissenschaftlichen Erkenntnisse gut in den Kontext erlebter Politik und belegen diese, zum anderen bieten sie, wie auch die grundsätzliche Frage der Studie nach den Auswirkungen der jeweiligen Karrierewege auf die politische Arbeit, Ansatzpunkte für neue Forschungen. Denn während diese Frage nun für Seiteneinsteiger zumindest teilweise beantwortet ist, drängt sie sich für andere Gruppen wie etwa die Karrierepolitiker geradezu auf - das Konzept der vorliegenden Studie ist also aufgegangen. 